\title{
Análise multidimensional da qualidade de vida nos municípios do Rio Grande do Sul (2010)
}

\author{
Multidimensional analysis of quality of life in the municipalities of Rio Grande do Sul
}

(2010)

\author{
Rayssa Vieira Kruger', Sibele Vasconcelos de Oliveira Vasconcelos de Oliveira", Priscila \\ Soares dos Santos"II
}

\begin{abstract}
Resumo
As abordagens contemporâneas sobre o fenômeno do desenvolvimento tendem a priorizar recortes analíticos multidimensionais. Em especial, os esforços em mensuração vão ao encontro da apropriação das diversas esferas do bem-estar, a incluir os aspectos culturais, econômicos, ambientais, entre outros. Destarte, o presente estudo propõe-se a analisar a qualidade de vida no estado do Rio Grande do Sul, a partir da operacionalização de ferramentais econométricos. Em suma, foi estimado o Índice de Qualidade de Vida (IQV) para o ano de 2010, cuja composição inclui 4 dimensões e 17 indicadores. Dentre os principais resultados da pesquisa, observou-se que poucos municípios atingiram elevado IQV, cujas localizações são dispersas no território gaúcho. A média do IQV para o estado foi de 0,307 , com o coeficiente de variação de $18 \%$. Os municípios com menor e maior IQV foram Passa Sete $(0,165)$ e Porto Alegre $(0,667)$, respectivamente. As Análises Fatorial e Exploratória de Dados Espaciais permitiram a constituição de três clusters padrão Alto - Alto e três clusters padrão Baixo - Baixo, mostrando que o estado possui regiões que carecem de ações que promovam o desenvolvimento. Dentre os aspectos a serem priorizados pela rede de proteção social, estão a saúde, educação e condições de moradias.
\end{abstract}

Palavras-chave: Desenvolvimento; Análise multidimensional; Bem-estar social

\begin{abstract}
Contemporary approaches to the phenomenon of development tend to prioritize multidimensional analytical clippings. In particular, efforts to measure meet the appropriation of the various spheres of well-being, including cultural, economic and environmental aspects, among others. Thus, this study aims to analyze the quality of life in the state of Rio Grande do Sul, from the operationalization of econometric tools. In summary, the Quality of Life Index (IQV) for 2010 was estimated, which comprises 4 dimensions and 17 indicators. Among the main results of the research, it was observed that few municipalities reached high IQV, whose locations are dispersed in the gaucho territory. The average IQV for the state was 0.307 , with a coefficient of variation of $18 \%$. The municipalities with the lowest and highest IQV were Passa Sete (0.165) and Porto Alegre (0.667), respectively. Factorial and Exploratory Spatial Data Analysis allowed the constitution of three standard High - High clusters and three standard Low - Low clusters, showing that the state has regions that lack actions that promote development. Among the aspects to be prioritized by the social safety net are health, education and housing conditions.
\end{abstract}

Keywords: Development; Multidimensional analysis; Social welfare.

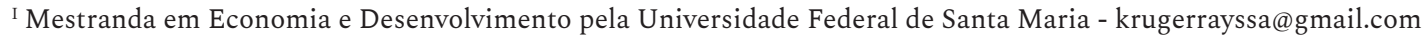

${ }^{\text {II }}$ Professora do Departamento de Economia e Relações Internacionais pela Universidade Federal de Santa Maria - sibele.ufsm@gmail.com

${ }^{\text {III } M e s t r a n d a ~ e m ~ E c o n o m i a ~ e ~ D e s e n v o l v i m e n t o ~ p e l a ~ U n i v e r s i d a d e ~ F e d e r a l ~ d e ~ S a n t a ~ M a r i a ~-~ p r i . s o a a r e s @ g m a i l . c o m ~}$
} 


\section{Introdução}

O debate sobre as estratégias para o desenvolvimento ocupa posição de destaque tanto na agenda científica quanto no policy making. São recorrentes as discussões que abarcam o entendimento acerca dos condicionantes do estado de bem-estar social, assim como a reflexão sobre os limites da ação pública para o enfrentamento às múltiplas privações que acometem - especialmente - as populações mais vulneráveis.

Sobretudo, as perspectivas interdisciplinar e multidimensional a respeito dos fenômenos correlatos ao desenvolvimento ganharam vulto nas últimas décadas. Os esforços em análise e mensuração contemplam exercícios de manipulação de dados empíricos, formulações teórico-metodológicas de caráter plural e diverso.

Nesse sentido, a presente pesquisa visa contribuir com a avaliação da qualidade de vida, a partir da operacionalização de informações estatísticas sobre a realidade socioeconômica do estado do Rio Grande do Sul (RS). Busca-se realizar uma análise multidisciplinar, de forma a incorporar a observação do status de vida da população gaúcha no que se refere às condições de moradia, acesso à saúde, educação, trabalho e renda.

Por meio da análise fatorial, é possível construir um Índice de Qualidade de Vida (IQV). Adicionalmente, a análise exploratória espacial permitirá identificar padrões de distribuição e associação dos IQVs nas diferentes regiões do Rio Grande do Sul. Logo, os resultados do presente exercício acadêmico são importantes, na medida em que revelam dinâmicas de concentração/dispersão da qualidade de vida dos municípios gaúchos.

Assim sendo, problematiza-se a trajetória ascendente das desigualdades intermunicipais no Rio Grande do Sul, intensificada durante os anos 1980 e 1990 e persistente nos dias atuais. Conforme destaca Fiori (2012), quando são avaliados os coeficientes de Williamson ${ }^{1}$, percebe-se que o padrão de distribuição intermunicipal do Produto Interno Bruto per capita no RS é bastante desigual. Se na década perdida e na década 1990 o estado gaúcho foi fortemente impactado pelas políticas macroeconômicas (que comprometeram o desempenho de atividades geradoras de trabalho e renda - tais como as agrícolas e industriais), nos períodos contemporâneos infere-se sobre as externalidades negativas do potencial processo de desindustrialização sobre a capacidade das regiões em promover desenvolvimento econômico.

Ao avaliarem impactos de fatores demográficos, fundiários e produtivos sobre o Índice de Desenvolvimento Humano dos municípios, Waquil e Filippi (2008) também concluíram sobre as diferenciadas dinâmicas regionais no estado do Rio Grande do Sul. Por conseguinte, percebe-se que explorar as fontes de heterogeneidades faz parte do processo de desmistificação da divisão do estado em duas metades territoriais bem definidas, a saber, a metade Sul e a metade Norte.

Tendo em vista o exposto, cabe mencionar que a redação está organizada em seis seções, a contar desta breve introdução. A seguir, apresenta-se o caráter multidimensional da qualidade de vida, bem como os esforços científicos em mensuração do fenômeno. Isto posto, são explorados os procedimentos metodológicos. A análise e discussão dos resultados compreendem a quarta seção do artigo. Por fim, são expostas as principais considerações do estudo e as referências consultadas ao longo do desenvolvimento do trabalho.

\section{O caráter multidimensional da qualidade de vida}

No campo das Ciências Econômicas, o debate sobre o desenvolvimento econômico esteve, por muito tempo, atrelado à descrição das dinâmicas de oscilação de produto e da renda per capita. No entanto, esse conceito foi mudando ao longo do tempo. Myrdal (1956), por exemplo, fez duras críticas à priorização do crescimento econômico nas estratégias nacionais de desenvolvimento, pois - sob sua concepção - não bastava agentes públicos e/ou privados estimularem o crescimento sem promover mudanças sociais e políticas para melhoria do bem-estar da população.

Frente ao embate conceitual entre crescimento e desenvolvimento econômico, Vasconcellos e Garcia (1998) argumentam que o crescimento econômico incorre em desenvolvimento econômico desde que esteja acompanhado de melhorias na qualidade de vida da população. Por sua vez, Sen (2001), apesar de reconhecer o papel crucial do crescimento de renda e da acumulação da riqueza na determinação do bem-estar social, afirma que "o desenvolvimento tem de estar relacionado sobretudo com a melhora da vida que levamos e das liberdades que desfrutamos" (SEN, 2001, p. 29).

Ainda, é possível perceber que muitas abordagens acerca do bem-estar e qualidade de vida estiveram a cargo de uma teoria baseada na satisfação e preferências. Contudo, conforme explicam Hausman, McPherson e Satz (2006), essa visão possui muitas limitações, visto que as pessoas podem ter falsas preferências e também porque essas preferências podem mudar com o transcorrer do tempo. Daí decorre que a definição do conceito de qualidade de vida

1 Indicador que representa a intensidade com que os PIBs per capita municipais se distanciam da média estadual.Quanto mais próximo de zero for o coeficiente de Williamson, mais homogênea é a distribuição intermunicipal (FIORI, 2012, p. 7). 
é um processo complexo, pois as pessoas podem valorizar realizações que não estejam propriamente relacionadas como o aumento de renda ou crescimento econômico.

Ao analisarem o perfil das pesquisas norte-americanas sobre o tema, Pereira, Teixeira e Santos (2012, p. 242) identificaram que, em um primeiro momento, "os indicadores econômicos foram o parâmetro primordial para se avaliar a qualidade de vida". Posteriormente, indicadores sociais passaram a ser incluídos no conjunto de fatores representantes do fenômeno. Para os autores:

Isso se deve, em parte, ao fato de que com uma ascensão econômica do país, problemas como violência e criminalidade emergiram apesar da riqueza econômica. Assim, os pesquisadores passaram a considerar indicadores sociais como críticos para definir o bem estar da nação. No entanto, as informações de cunho social, coletadas nessa época, se focalizavam apenas em fatores externos, presumidamente determinantes da qualidade de vida, como instrução, renda e moradia (Day \& Jankey, 1996), como utilizados, ainda hoje, em larga escala (PEREIRA; TEIXEIRA; SANTOS, 2012, p. 242).

Outrossim, a qualidade de vida pode estar associada a múltiplos fatores, tais como conhecimento, saúde, meio ambiente, liberdade política e demais aspectos da vida que não dependem exclusivamente da renda (UL HAQ, 1995). Especialmente, Seidl e Zannon (2004) reforçam a tese de que existem dois aspectos relevantes a serem considerados quando da avaliação da qualidade de vida, a saber, a subjetividade e multidimensionalidade.

No que concerne à subjetividade, trata-se de considerar a percepção da pessoa sobre o seu estado de saúde e sobre os aspectos não-médicos do seu contexto de vida. Em outras palavras, como o indivíduo avalia a sua situação pessoal em cada uma das dimensões relacionadas à qualidade de vida. [...] O consenso quanto à multidimensionalidade refere-se ao reconhecimento de que o construto é composto por diferentes dimensões (SEIDL; ZANNON, 2004, p. 582).

Nussbaum e Sen (1993) avaliam a qualidade de vida a partir das chamadas capacitações (que representam as possíveis combinações de coisas que uma pessoa está apta a fazer ou ser) e as funcionalidades (que representam as coisas que uma pessoa de fato faz ou é). Desta forma, a capacitação acaba sendo um reflexo das combinações alternativas de funcionalidades que u0ma pessoa pode adquirir. Sendo assim, a qualidade de vida pode ser analisada através dessas funcionalidades, a incluir as "elementares" (como, por exemplo, ter saúde e nutrir-se) ou "não-elementares" (em exemplo, participar da integração social).

Neste sentido, as capacitações são necessárias ao processo de expansão da qualidade de vida. Conforme explica Amartya Sen (2001), a capacitação de uma pessoa dependerá de um conjunto de fatores e arranjos sociais. É mensurada pelo conjunto de oportunidades reais que ela tem em seu favor, e não necessariamente pelas realizações efetivas da pessoa. Portanto, a qualidade de vida não deve ser entendida como um mero conjunto de bens e serviços, mas como as oportunidades efetivas das quais as pessoas dispõem para ser.

Ao reconhecer que a qualidade de vida é uma noção humana, Minayo, Hartz e Buss (2000, p. 8) inferem que o conceito está atrelado à capacidade dos seres humanos em "efetuar uma síntese cultural de todos os elementos que determinada sociedade considera seu padrão de conforto e bem-estar". De acordo com os supracitados autores, sua compreensão passa pela apropriação ao "grau de satisfação encontrado na vida familiar, amorosa, social e ambiental e à própria estética existencial”.

Quadro 1 - Fóruns de referência ao conceito de qualidade de vida

\begin{tabular}{l|c}
\hline \multicolumn{1}{c|}{$\begin{array}{c}\text { Relatividade da noção } \\
\text { no âmbito individual }\end{array}$} & Especificidades \\
\hline Histórica & $\begin{array}{c}\text { Os parâmetros de qualidade de vida diferem de acordo } \\
\text { com as dinâmicas de desenvolvimento econômico, social } \\
\text { e tecnológico observados em uma mesma sociedade. }\end{array}$ \\
Cultural & $\begin{array}{c}\text { As tradições dos povos definem os valores e } \\
\text { necessidades que são construídos e hierarquizados. } \\
\text { Vinculada ao bem-estar das camadas superiores e à } \\
\text { passagem de um limiar a outro. }\end{array}$ \\
\hline
\end{tabular}

Fonte: Minayo, Hartz e Buss (2000, p. 9). 
Palomba (2002) destaca que a qualidade de vida está relacionada com: (i) fatores materiais, associados à renda disponível, posição no mercado de trabalho, saúde, nível de educação entre outros; (ii) fatores ambientais, tais como acesso aos serviços básicos, níveis de segurança, acesso à tecnologia e características de moradia; (iii) fatores de relacionamentos, ou seja, relações com familiares, amigos e conexões sociais em geral; e (iv) políticas governamentais, pois a qualidade de vida não é relacionada apenas à perspectiva dos indivíduos, mas também à perspectiva social, de modo que depende das políticas públicas existentes.

Desse modo, é possível observar o caráter multifacetado que possui o fenômeno da qualidade de vida.

As abordagens gerais ou holísticas baseiam-se na premissa segundo a qual o conceito de qualidade de vida é multidimensional, apresenta uma organização complexa e dinâmica dos seus componentes, difere de pessoa para pessoa de acordo com seu ambiente/contexto e mesmo entre duas pessoas inseridas em um contexto similar. Características como valores, inteligência, interesses são importantes de serem considerados (PEREIRA; TEIXEIRA; SANTOS, 2012, p. 243).

Ressalta-se que a determinação das dimensões que são valoradas pelos indivíduos na constituição da qualidade de vida é muito complexo e mensurá-las apresenta-se como um desafio aos cientistas sociais. Sobretudo, foi nas décadas de 1970 e 1980 que surgiram os primeiros "indicadores multidimensionais de qualidade de vida", com o objetivo de dar uma resposta à crescente insatisfação gerada pelos indicadores tradicionais de renda (VÁZQUEZ et al., 2013).

Para Schneider e Freitas (2013), desde a década de 1990, o Índice de Desenvolvimento Humano (IDH) ${ }^{2}$ vem referenciando parte expressiva das análises comparativas, além das decisões de agentes públicos no tocante aos fenômenos do desenvolvimento e qualidade de vida. No entanto, as críticas ao IDH tem cunho metodológico, em termos de unidade de análise, variáveis amostradas e técnicas de agregação.

Sendo assim, Freitas (2015, p. 58) sugere que os indicadores para representação da qualidade de vida devem ser "heterogêneos, multidimensionais e relacionados com a realidade". A autora sintetiza algumas dimensões empregadas no âmbito das ciências sociais para mensurar qualidade e/ou condições de vida, isto é, as denominadas necessidades sociais, materiais e de crescimento pessoal. Todas essas são passíveis de serem avaliadas com base em indicadores subjetivos e objetivos (vide Figura 1)

Figura 1 - Indicadores objetivos e subjetivos da qualidade de vida

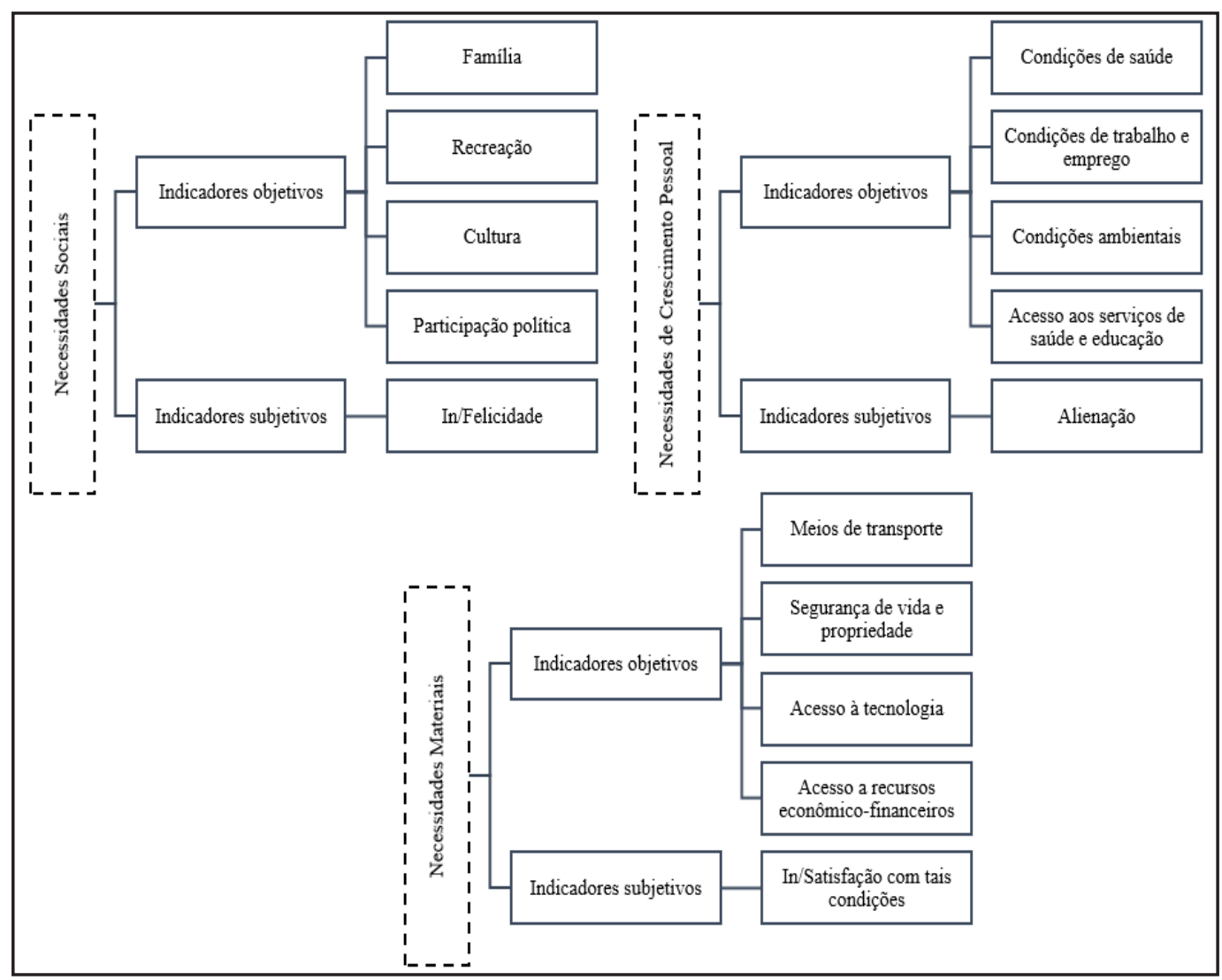

Fonte: Adaptado de Freitas (2015) e Schneider e Freitas (2013)

2- O IDH é um indicador de qualidade de vida e trabalha em três pilares: i) Renda, ii) Longevidade e iii) Educação. O índice varia de 0 a 1 , sendo que quando o IDH for menor ou igual a 0,5 , é classificado como baixo desenvolvimento. Os valores entre 0,5 e 0,8 , correspondem ao médio desenvolvimento e, por fim, valores maiores que 0,8 , representam o alto desenvolvimento humano (PAULANI; BRAGA, 2007). 
A seguir, discorre-se sobre as características do desenvolvimento e qualidade de vida no estado do Rio Grande do Sul, de forma a contemplar as diferentes facetas dos fenômenos. A análise é realizada por meio de revisão de literatura e exploração de dados estatísticos para a região.

\section{Insights sobre qualidade de vida e desigualdades regionais no Rio Grande do Sul}

Os estudos acerca da formação socioeconômica do estado do Rio Grande do Sul apontam para a existência de determinada divisão regional, cujas implicações são percebidas quando são avaliados os desequilíbrios em termos de níveis de desenvolvimento e qualidade de vida da população. Em particular, muitas pesquisas evidenciam o atraso econômico da região Sul do estado gaúcho, que é justificado pela especialização produtiva agropecuária e pela presença expressiva de latifúndios (ALONSO; BENETTI; BANDEIRA, 1994).

Após revisão de literatura, Arend e Cario (2010, p. 384) concluem sobre as dinâmicas de desenvolvimento da região Sul-Riograndense, que foram diretamente impactadas pelos efeitos econômicos limitados a longo prazo da agricultura de plantation:

As grandes propriedades restringiriam o poder de crescimento econômico da região pelo surgimento de retornos decrescentes da atividade principal, pois a concentração tenderia a levar uma parcela considerável da população à exclusão da economia de mercado. A distribuição de renda desigual faria com que a parcela excluída da economia de mercado gastasse a maior parcela de sua renda com gêneros de primeira necessidade. Por outro lado, os latifundiários tenderiam a gastar seus rendimentos em importações de bens de luxo. Assim, a produção de manufaturados na região ficaria restringida e a região, a longo prazo, estagnaria (AREND; CARIO, 2010, p. 384).

É interessante observar que as diferenças entre as estruturas econômicas consolidadas nas áreas ao norte e ao sul do estado dominaram a pauta de investigação histórica dos padrões de desigualdade na região. Contudo, o vasto número de pesquisas sobre as desigualdades regionais gaúchas poucas vezes abordou multidimensionalmente o fenômeno do desenvolvimento, tais como a observação de fatores educacionais, de saúde, moradia, entre outros (BERNARDINI; KANG; WINK JR., 2015). Logo, faz-se necessária tal reflexão, com o intuito de contribuir para a compreensão das realidades vivenciadas pela população gaúcha.

Cabe mencionar que o Rio Grande do Sul possui aproximadamente $282 \mathrm{mil} \mathrm{km}^{2}$ e elevada concentração das atividades econômicas. No início do século XXI, mais da metade do PIB e $64 \%$ da produção industrial eram desenvolvidos nas regiões do Vale do Rio dos Sinos, da Serra e Metropolitana. As mesmas regiões aglutinavam $42 \%$ da população gaúcha, a despeito de representarem somente 5,24\% do território estadual (OLIVEIRA, 2005).

Para Oliveira (2005, p. 96), o padrão de organização das atividades econômicas no Rio Grande do Sul explica os desequilíbrios regionais em termos de distribuição da renda e riqueza. Reconhecido por seus pólos "o coureiro-calçadista no Vale do Rio dos Sinos, autopeças e moveleiro na Serra, máquinas e implementos agrícolas na Fronteira Noroeste, conservas no Sul", o estado encontra dificuldades para socializar os ganhos do sistema econômico e, por consequência, para promover qualidade de vida à sua população.

Ao passo que as disparidades regionais tem se ampliado ao longo das últimas décadas, Conterato, Schneider e Waquil (2007, p. 164) evidenciam que a "existência de estruturas econômicas cristalizadas nas áreas mais pobres acabam por impedir que forças sociais e econômicas mais dinâmicas conduzam essas economias regionais para a rota da expansão e do desenvolvimento econômico regional mais autônomo". Neste sentido, a avaliação do Índice de Desenvolvimento Socioeconômico (IDESE) ${ }^{3}$ permitiu à Bernardini, Kang e Wink Jr (2015) identificar a presença de desigualdades em várias dimensões do bem-estar, especialmente percebidas nas regiões mais a norte e nordeste vis-à-vis ao sul do estado.

Segundo os autores, "as desigualdades regionais gaúchas não se confinam apenas às alardeadas diferenças entre norte, nordeste e sul, mas a capital e os arredores, com seus problemas de pobreza e exclusão urbana" (BERNARDINI; KANG; WINK JR., 2015, p. 67). As marcantes disparidades nas regiões mais urbanizadas do RS demonstram diferenciais importantes de renda, no acesso à saúde e educação.

À título de conhecimento, destaca-se que a mesorregião mais urbanizada do estado é a Metropolitana de Porto Alegre e a com menor taxa de urbanização é a Centro Oriental Rio-grandense. Durante o período de 2000 a 2010, a mesorregião que presenciou o maior crescimento das taxas de urbanização foi a Noroeste Rio-grandense. O acesso aos dados estatísticos para o ano de 2010 revela também que 16,51\% dos assalariados da mesorregião Noroeste

3 O IDESE realiza a avaliação de 12 indicadores (quantitativos e qualitativos) que buscam retratar a situação socioeconômica dos municípios gaúchos quanto aos diferentes aspectos do desenvolvimento, a saber, educação, renda e saúde (FEE, 2018). 
Rio-grandense recebiam até um salário mínimo, enquanto que na região Sudeste Rio-grandense o percentual é de $31,46 \%$ (IBGE, 2019).

Tabela 1 - Percentual de pessoas por classes de rendimento nominal mensal (2010)*

\begin{tabular}{|c|c|c|c|c|c|c|c|}
\hline Mesorregião Geográfica & $\begin{array}{c}\text { Até } 1 \\
\text { salário } \\
\text { mínimo }\end{array}$ & $\begin{array}{c}\text { Mais } \\
\text { de } 1 \text { a } 2 \\
\text { salários } \\
\text { mínimos }\end{array}$ & $\begin{array}{l}\text { Mais } \\
\text { de } 2 \text { a } 3 \\
\text { salários } \\
\text { mínimos }\end{array}$ & $\begin{array}{l}\text { Mais } \\
\text { de } 3 \text { a } 5 \\
\text { salários } \\
\text { mínimos }\end{array}$ & $\begin{array}{c}\text { Mais de } \\
5 \text { a } 10 \\
\text { salários } \\
\text { mínimos }\end{array}$ & $\begin{array}{c}\text { Mais de } \\
10 \text { a 20 } \\
\text { salários } \\
\text { mínimos }\end{array}$ & $\begin{array}{l}\text { Mais } \\
\text { de } 20\end{array}$ \\
\hline Noroeste Rio-grandense & 29,41 & 25,34 & 8,81 & 6,76 & 4,12 & 1,29 & 0,55 \\
\hline Nordeste Rio-grandense & 16,51 & 29,23 & 13,43 & 10,58 & 6,55 & 1,79 & 0,73 \\
\hline $\begin{array}{l}\text { Centro Ocidental Rio- } \\
\text { grandense }\end{array}$ & 28,3 & 22,12 & 7,68 & 6,66 & 5,24 & 1,96 & 0,77 \\
\hline $\begin{array}{l}\text { Centro Oriental Rio- } \\
\text { grandense }\end{array}$ & 26,57 & 29,80 & 9,61 & 7,29 & 4,11 & 1,11 & 0,47 \\
\hline $\begin{array}{l}\text { Metropolitana de Porto } \\
\text { Alegre }\end{array}$ & 18,92 & 27,42 & 10,2 & 8,17 & 5,97 & 2.35 & 1,08 \\
\hline Sudoeste Rio-grandense & 31,46 & 21,5 & 6,25 & 5,15 & 3,38 & 1,16 & 0,51 \\
\hline Sudeste Rio-grandense & 29,99 & 22,53 & 7,46 & 5,88 & 3,94 & 1,28 & 0,48 \\
\hline
\end{tabular}
Fonte: IBGE (2019).

*Salário mínimo de $\mathrm{R} \$ 510,00$.

Ao avaliarem os diferenciais de crescimento entre as Regiões Norte, Sul e Nordeste do RS Marquetti, Bérni e Marques (2005) concluíram que a especialização setorial e a acumulação de capital são fundamentais para justificar os resultados regionais ímpares. Os referidos resultados foram obtidos por meio de instrumentos econométricos e manipulação de dados para os anos 1990. O comportamento díspar da distribuição da renda per capita entre as mesorregiões também é percebido quando são avaliados os diferentes indicadores de desenvolvimento.

A tabela 2 apresenta o Índice de Desenvolvimento Socioeconômico do Rio Grande do Sul para o período de 2007 a 2016. De forma similar ao Índice de Desenvolvimento Humano (IDH), os resultados do IDESE podem ser estratificados. Em síntese, considera-se que há baixo desenvolvimento para valores até 0,499. Médio e alto desenvolvimento são definidos nos intervalos entre “0,500 - 0,799” e “0,800 - 1,00”, respectivamente (OLIVEIRA, 2013).

Tabela 2 - Índice de Desenvolvimento Socioeconômico do Rio Grande do Sul (2007 - 2016) ${ }^{4}$

\begin{tabular}{|c|c|c|c|c|c|c|c|c|c|c|}
\hline \multirow{2}{*}{ Mesorregiões } & \multicolumn{10}{|c|}{ IDESE } \\
\hline & 2007 & 2008 & 2009 & 2010 & 2011 & 2012 & 2013 & 2014 & 2015 & 2016 \\
\hline Centro Ocidental Rio-Grandense & 0,67 & 0,68 & 0,68 & 0,69 & 0,71 & 0,71 & 0,73 & 0,75 & 0,74 & 0,75 \\
\hline Centro Oriental Rio-Grandense & 0,69 & 0,70 & 0,71 & 0,72 & 0,73 & 0,74 & 0,76 & 0,77 & 0,75 & 0,76 \\
\hline Metropolitana de Porto Alegre & 0,71 & 0,71 & 0,72 & 0,73 & 0,73 & 0,74 & 0,75 & 0,76 & 0,75 & 0,75 \\
\hline Nordeste Rio-Grandense & 0,74 & 0,74 & 0,75 & 0,77 & 0,78 & 0,79 & 0,80 & 0,81 & 0,80 & 0,80 \\
\hline Noroeste Rio-Grandense & 0,69 & 0,70 & 0,71 & 0,72 & 0,74 & 0,74 & 0,77 & 0,78 & 0,77 & 0,77 \\
\hline Sudeste Rio-Grandense & 0,64 & 0,64 & 0,65 & 0,66 & 0,66 & 0,67 & 0,68 & 0,69 & 0,69 & 0,70 \\
\hline Sudoeste Rio-Grandense & 0,63 & 0,64 & 0,65 & 0,65 & 0,66 & 0,67 & 0,69 & 0,70 & 0,70 & 0,70 \\
\hline
\end{tabular}

Fonte: FEE (2018).

4 O início do período de análise é 2007, por conta da disponibilidade de informações estatísticas divulgadas pela FEE (2018). 
Neste ínterim, percebe-se que somente a mesorregião Nordeste Rio-Grandense galgou alto desenvolvimento socioeconômico. Os piores IDESE foram experienciados pelas regiões Sudeste e Sudoeste Rio-Grandense. A despeito dos avanços dos anos mais recentes, muitos são os desafios do estado do Rio Grande do Sul na promoção da qualidade de vida à população. A concentração de renda permanece expressiva (o índice de Gini era de 0,58 em 2000; 0,54 em 2010 e 0,49 em 2017) e a renda per capita dos vulneráveis à pobreza decaiu nos últimos anos (era de $\mathrm{R} \$ 144,55$ em 2000 , caindo para $\mathrm{R} \$ 143,57$ em 2017). Destarte, explora-se a seguir um conjunto de indicadores não avaliados pelos índices tradicionalmente estimados por agências de pesquisa e órgãos institucionais, mas que podem contribuir para o entendimento acerca das dinâmicas de vida e bem-estar usufruídos pela população do Rio Grande do Sul.

\section{Procedimentos metodológicos}

A presente seção dedica-se a descrever os procedimentos metodológicos adotados para construção do Índice de Qualidade de Vida (IQV) para os municípios do Rio Grande do Sul, bem como para coleta e análise de dados estatísticos. Neste sentido, apresentam-se as técnicas econométricas em Análise Fatorial e Análise Exploratória de Dados Espaciais (AEDE).

Para a execução da primeira etapa do estudo, ou seja, a elaboração do IQV, foram delimitadas as dimensões e variáveis de avaliação do fenômeno da qualidade de vida. Após revisão de literatura, foi possível elencar para análise 17 indicadores, organizados em 4 dimensões analíticas: trabalho e renda, moradia, saúde e educação. A seleção foi realizada com base na disponibilidade de dados secundários para o Rio Grande do Sul. Desse modo, foram coletados dados secundários divulgados pelo Instituto Brasileiro de Geografia e Estatística (IBGE), Atlas de Desenvolvimento Humano (ADH), Instituto Nacional de Estudos e Pesquisas Educacionais Anísio Teixeira (INEP) e Fundação de Economia e Estatística (FEE).

O Quadro 2 ilustra as variáveis selecionadas para compor o IQV e as respectivas fontes de dados empíricos para o estado gaúcho no ano de 2010.

Quadro 2 - Descrição das variáveis utilizadas e suas respectivas fontes

\begin{tabular}{|c|c|c|}
\hline Dimensão & Variável & Fonte \\
\hline \multirow{6}{*}{ Moradia } & \multirow{6}{*}{$\begin{array}{c}\text { Domicílios com microcomputador (com acesso à internet) } \\
\text { Domicílios com geladeira } \\
\text { Domicílios com máquina de lavar } \\
\text { Domicílios com automóvel para uso particular } \\
\text { Porcentagem da população em domicílios com banheiro e água } \\
\text { encanada } \\
\text { Porcentagem da população em domicílios com coleta de lixo } \\
\text { Porcentagem da população em domicílios com densidade maior que }\end{array}$} & IBGE \\
\hline & & IBGE \\
\hline & & IBGE \\
\hline & & $\mathrm{ADH}$ \\
\hline & & $\mathrm{ADH}$ \\
\hline & & $\mathrm{ADH}$ \\
\hline \multirow{2}{*}{ Saúde } & Taxa de mortalidade infantil & $\mathrm{ADH}$ \\
\hline & Esperança de vida ao nascer & $\mathrm{ADH}$ \\
\hline \multirow{5}{*}{ Educação } & \multirow{5}{*}{$\begin{array}{c}\text { Taxa de alfabetização (\%) } \\
\text { Matrículas no ensino regular } \\
\text { Estabelecimentos de ensino } \\
\text { Percentual de pessoas com } 18 \text { anos ou mais com fundamental } \\
\text { completo } \\
\text { Percentual de pessoas com de } 18 \text { anos ou mais com médio completo } \\
\text { Percentual de pessoas com de } 25 \text { anos ou mais com superior }\end{array}$} & $\mathrm{IBGE}$ \\
\hline & & INEP \\
\hline & & $\mathrm{ADH}$ \\
\hline & & $\mathrm{ADH}$ \\
\hline & & $\mathrm{ADH}$ \\
\hline \multirow[t]{2}{*}{$\begin{array}{l}\text { Renda e } \\
\text { emprego }\end{array}$} & $\begin{array}{l}\text { Rendimento nominal médio mensal per capita dos domicílios } \\
\text { particulares permanentes }\end{array}$ & IBGE \\
\hline & Número de vínculos empregatícios & FEE \\
\hline
\end{tabular}


A estimação do IQV foi obtida por meio Análise Fatorial (AF). Segundo Mingoti (2005), esse instrumento de análise tem como objetivo agrupar as variáveis originais em subconjuntos de novas variáveis não correlacionadas, ou seja, reduzir as principais informações das variáveis originais (MINGOTI, 2005). Formalmente é dado por:

$$
X_{i}=a_{i 1} F_{1}+a_{i 2} F_{2}+
$$

em que: $\boldsymbol{X}_{\mathbf{i}}$ representam as variáveis descritas no Quadro $1 ; \boldsymbol{a}^{-}-$ano as cargas fatoriais est $^{\cdot}$ adas, que expressam o grau de relacionamento entre os fatores e as variáveis; $\boldsymbol{F}_{\mathbf{1}}, \boldsymbol{\cdots}, \boldsymbol{F} \mathbf{\boldsymbol { Y }}$ são os fatores comuns; e $\mathbf{E}_{\mathbf{i}}$ é o termo de erro.

No presente estudo, as cargas fatoriais foram estimadas pelo método dos componentes principais, isto é, o primeiro fator tem o maior poder de explicação e assim sucessivamente (MINGOTI, 2005). Para a rotação ortogonal dos fatores, foi empregado o método varimax. Segundo (MINGOTI, 2005), esse é o método mais utilizado na literatura acadêmica, pois possibilita que os coeficientes de correlação entre as variáveis e os fatores fiquem o mais próximo possível de zero ou um.

Identificados os fatores, a próxima etapa da Análise Fatorial é desenvolvida com base na estimação dos escores fatoriais, dados formalmente por:

$$
\hat{F}_{j k}=w_{j 1} X_{1 i}+w_{j 2} X_{2 i}+\cdots+w_{j p} Z_{p i}
$$

em qua $\boldsymbol{Y}_{\mathbf{n}} \boldsymbol{X}_{\mathbf{A}}$.... $\boldsymbol{X}$; cão os valores observados das variáveis $\boldsymbol{X}_{\mathbf{i}}$ para o k-ésimo elemento amostral e os coeficiente $\boldsymbol{w}_{\tilde{j}}, \bar{i}=\mathbf{1} \mathbf{Z} \cdot \boldsymbol{- \mu} \boldsymbol{p}$ são os pesos de ponderação de cada variável no fator $\boldsymbol{F}_{\mathbf{J}}$ ” (MINGOTI, 2005, p. 116).

De posse dos escores fatoriais, obtidos a partir da equação (2), e dos resultados do estudo de Ferreira et al. (2016), pode-se construir o IQV (vide equação 3).

$$
I Q V_{m}=\sum_{j=1}^{p}\left(\frac{\sigma_{j}^{2}}{\sum_{j=1}^{p} \sigma_{j}^{2}} F_{j m}\right)
$$

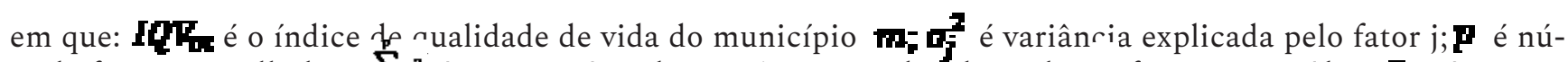
mero de fatores escolhidos; $\sum_{\boldsymbol{g}}^{\mathbf{\sigma}} \mathbf{j}$ é o somatório das variâncias explicadas pelos $\boldsymbol{P}$ fatores extraídos; $\boldsymbol{F}_{\boldsymbol{m}}$ é escore

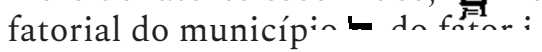

Para obter. Is dos escores originais, adotou-se o seguinte procedimento:

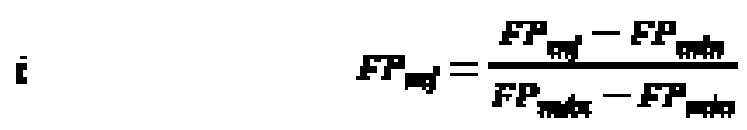

em que: $\boldsymbol{F P}_{\mathbf{r i}}$ é

do j-ésimo escore fatorial observado e $\boldsymbol{F} \boldsymbol{P}$ é o maior valor do j-ésimo escore fatorial observado.

Desse modo, o IQV varia entre os valores 0 e 1, sendo que quanto mais próximo de 1, melhor é a Qualidade de Vida.

$\begin{array}{cc}\text { Baixa } & \text { Alta } \\ \text { Qualidade de Vida } & \text { Qualidade de Vida }\end{array}$

Para a validação do modelo fatorial, foram realizados os testes de Kaiser-Meyer-Olkin (KMO) e o Barlett Test of Sphericity (BTS). O KMO pode variar entre 0 e 1 e tem como objetivo testar se a AF é apropriada para o estudo. Mingoti (2005) recomenda que o resultado do referido teste seja acima de 0,7 e, preferencialmente, igual ou maior que 0,8 para que a base de dados esteja apropriada para estudar o fenômeno em questão.

Já o BTS possui o objetivo de verificar se a matriz de correlações é igual à uma matriz identidade, testando as seguintes hipóteses:

$$
\begin{aligned}
& H_{0}=P_{m p}=I_{p q} \text { e } \\
& H_{1}=R_{m p} \neq I_{10 p}
\end{aligned}
$$

em que: $\mathbf{P}_{\mathbf{m}}$ é a matriz de correlação e $\boldsymbol{I}_{\mathbf{m}}$ é a matriz identidade. Logo, para que os dados sejam adequados, deve-se rejeitar $\boldsymbol{H}_{\mathbf{D}}$ (MINGOTI, 2005). 
Para observar se o IQV de um determinado município possui o mesmo padrão que os municípios vizinhos, foi aplicada a análise exploratória de dados espaciais (AEDE). Essa consiste em um recurso da econometria espacial e que, segundo Almeida (2012), compreende uma "coleção de técnicas para descrever e visualizar distribuições espaciais, identificar localidades atípicas [...], descobrir padrões de associação espacial [...] e sugerir diferentes regimes espaciais" (ANSELIN, 1999, p. 258 apud ALMEIDA, 2012, p.102).

Um dos coeficientes de autocorrelação espacial existente no campo da AEDE é a Estatística I de Moran. Para o presente estudo, foi calculado o I de Moran Global Univariado e o I de Moran Local (LISA). O primeiro é dado por:

$$
I_{t}=\left(\frac{n}{s_{0}}\right)\left(\frac{z_{t}^{\prime} w z_{t}}{z_{t}^{\prime} z_{t}}\right)
$$

em que n representa o número de regiões; Z representa as variáveis padronizadas; $\boldsymbol{S}_{\mathbf{D}}$ é igual a operação $\boldsymbol{\Sigma}_{\mathbf{Z}} \mathbf{w}_{\mathbf{i j}}$ que significa que todos os elementos da matriz de pesos espaciais W devem ser somados; WZ denota os valores médios da variável em estudo padronizada nos vizinhos, definidos segundo uma matriz de ponderação espacial (W) (ALMEIDA, 2012).

A matriz de pesos espaciais (W) utilizada no presente estudo foi a rainha. Segundo Almeida (2012, p. 3), tendo em vista os movimentos das peças em um Tabuleiro de xadrez, a matriz de pesos espaciais é Rainha quando "além das fronteiras com extensão diferente de zero, puderem ser considerados os vértices como contíguos, na visualização de um mapa”.

O I de Moran tem um valor esperado de -[1/(n-1)], ou seja, o valor que seria obtido se não houvesse padrão espacial nos dados. Para observação do I de Moran, deve-se levar em consideração os coeficientes de correlação -1 e +1. Em outras palavras, quanto mais próximo de -1 a autocorrelação espacial é negativa, situação em que, quando as unidades espaciais obtiverem elevados, são rodeadas por unidades espaciais com z baixos. E quando I estiver próximo de +1 , a autocorrelação é positiva, sendo que o elemento espacial analisado será elevado (ALMEIDA, 2012).

Além do resultado do I de Moran, implementa-se uma abordagem complementar, denominada de diagrama de dispersão de Moran. Nesse diagrama, há quatro tipos de associação linear espacial (padrões) que podem ser observados: o padrão Alto-Alto (AA), Baixo-Baixo (BB), Alto-Baixo (AB) e Baixo-Alto (BA) (ALMEIDA, 2012). Por fim, o I de Moran Global possui as seguintes hipóteses a serem testadas:

$$
\begin{aligned}
& \boldsymbol{H}_{\mathbf{D}}=\text { Aleatoriedade espacial } \\
& \boldsymbol{H}_{\mathbf{2}}=\text { Autocorrelação espacial }
\end{aligned}
$$

Depois de ter verificar a presença da autocorrelação Global, deve-se verificar se há presença de autocorrelação local, procedimento implantado através do I de Moran Local Univariado, que é dado por (ALMEIDA, 2012):

$$
I_{i}=z_{i} \sum_{j=1}^{J} w_{i j} z_{j}
$$

em que: $\boldsymbol{I}_{\mathbf{i}}$ abrange apenas os vizinhos da observação $\mathbf{i}$, que são definidos conforme a matriz de pesos espaciais. Logo, para cada observação do estudo, é calculado um $\boldsymbol{I}_{\mathbf{i}}$.

Para a melhor visualização dos resultados, será elaborado o mapa de clusters LISA, que "combina a informacão do diagrama de dispersão de Moran e a informação do mapa de significância das medidas de associação local $\boldsymbol{J}_{\mathbf{z}}$ ” (ALMEIDA, 2012, p. 127). Nesse mapa, podem ser identificados os padrões espaciais dos clusters (AA, BB, AB, BA), de acordo com o resultado do I de Moran.

\section{Análise e discussão dos resultados}

A Análise Fatorial foi desenvolvida a partir da manipulação de dados secundários sobre a realidade socioeconômica do estado do Rio Grande do Sul, considerando a disponibilidade de informações para o ano de 2010. Para tanto, testou-se a adequabilidade dos dados para se aplicar o método quantitativo. Com base nisso, o teste KMO foi de 0,8360 e o BTS foi de $1.8 \mathrm{e}+04$ (com p-valor de 0.0000 ), demonstrando que os dados empregados na análise das 17 variáveis são adequados.

A Tabela 3 indica quatro fatores, com raiz característica maior que 1 e que sintetizam as informações das dezessete variáveis originais utilizadas no presente estudo. Observa-se que após rotação, os quatro fatores explicam $83 \%$ da variância total das variáveis. 
Tabela 3 - Fatores, raiz característica, variância explicada e acumulada

\begin{tabular}{c|c|c|c}
\hline Fatores & Raiz Característica & Variância Explicada & Variância Acumulada \\
\hline F1 & 6.04391 & 0.3555 & 0.3555 \\
F2 & 3.42979 & 0.2018 & 0.5573 \\
F3 & 2.48379 & 0.1461 & 0.7034 \\
F4 & 2.18319 & 0.1284 & 0.8318 \\
\hline
\end{tabular}

Fonte: Estimativas utilizando dados disponibilizados pelo ADH (2019), IBGE (2019), FEE (2019) e INEP (2019).

Por sua vez, a Tabela 4 apresenta os resultados da Análise Fatorial, com suas respectivas cargas fatoriais e comunalidades.

Tabela 4 - Cargas fatoriais e Comunalidades

\begin{tabular}{|c|c|c|c|c|c|}
\hline \multirow{2}{*}{$\begin{array}{c}\text { Variáveis } \\
\text { F1 }\end{array}$} & \multicolumn{4}{|c|}{ Cargas Fatoriais } & \multirow{2}{*}{ Comunalidades } \\
\hline & F2 & F3 & F4 & & \\
\hline \multirow{7}{*}{ Moradia } & 0.9836 & 0.1471 & 0.0402 & 0.0162 & 0.0090 \\
\hline & 0.9674 & 0.2398 & -0.0107 & 0.0466 & 0.0044 \\
\hline & 0.9760 & 0.2073 & 0.0108 & 0.0417 & 0.0026 \\
\hline & 0.9647 & 0.2503 & 0.0104 & 0.0450 & 0.0046 \\
\hline & -0.0041 & 0.2146 & 0.7537 & 0.1599 & 0.3604 \\
\hline & 0.0065 & 0.2584 & 0.2113 & 0.3210 & 0.7855 \\
\hline & 0.1059 & 0.2220 & -0.8545 & -0.0652 & 0.2051 \\
\hline \multirow{2}{*}{ Saúde } & -0.0433 & -0.1086 & -0.1470 & -0.9683 & 0.0271 \\
\hline & 0.0524 & 0.1350 & 0.2045 & 0.9536 & 0.0279 \\
\hline \multirow{6}{*}{ Educação } & 0.0455 & 0.3429 & 0.7252 & 0.2831 & 0.2743 \\
\hline & 0.9551 & 0.2549 & -0.0379 & 0.0566 & 0.0182 \\
\hline & 0.3200 & 0.6546 & -0.2345 & 0.1308 & 0.3969 \\
\hline & 0.1930 & 0.8830 & 0.2138 & 0.1691 & 0.1088 \\
\hline & 0.2328 & 0.8842 & 0.2660 & 0.1648 & 0.0661 \\
\hline & 0.3181 & 0.8059 & 0.1375 & 0.0423 & 0.2286 \\
\hline \multirow{2}{*}{$\begin{array}{l}\text { Renda e } \\
\text { Emprego }\end{array}$} & 0.2742 & 0.4673 & 0.5947 & 0.1980 & 0.3136 \\
\hline & 0.9783 & 0.1119 & 0.0653 & 0.0079 & 0.0261 \\
\hline
\end{tabular}

Fonte: Estimativas com base em dados disponibilizados pelo ADH (2019), IBGE (2019), FEE (2019) e INEP (2019).

Observa-se que foram extraídos 4 fatores e, para suas interpretações, foram consideradas as cargas fatoriais maiores ou igual a 0,6 . O primeiro fator está correlacionado positivamente com as variáveis (Domicílios com microcomputador com acesso à internet), (Domicílios com geladeira), (Domicílios com máquina de lavar), (Domicílios com automóvel para uso particular), (Matrículas no ensino regular), (Número de vínculos empregatícios). 
Logo, denominou-se esse primeiro fator de Indicador de acesso a Bens Duráveis.

O segundo fator está correlacionado positivamente com as variáveis (Estabelecimentos de ensino), (Percentual de pessoas com 18 anos ou mais com fundamental completo), (Percentual de pessoas com de 18 anos ou mais com médio completo), (Percentual de pessoas com de 25 anos ou mais com superior completo). Assim sendo, nomeou-o de Indicador Educacional.

O terceiro fator está correlacionado positivamente com as variáveis (Porcentagem da população em domicílios com banheiro e água encanada), (Taxa de alfabetização), (Rendimento nominal médio mensal per capita dos domicílios particulares permanentes). Ainda, esteve correlacionado negativamente com (Porcentagem da população em domicílios com densidade maior que 2). Desse modo, pode-se nomeá-lo enquanto fator Indicador da capacidade econômica de ter melhores condições de moradia.

O último fator estimado está fortemente correlacionado negativamente com a variável (Taxa de mortalidade infantil) e positivamente com (Esperança de vida ao nascer). Com base nisso, denominou-o como Indicador de condições de saúde. Com base na estimação das referidas cargas fatoriais, construiu-se o IQV por meio dos escores fatoriais de cada município.

A média do IQV para o estado do Rio Grande do Sul em 2010 foi de 0,307 e o coeficiente de variação foi de $18 \%$, apresentando média dispersão do índice e certa heterogeneidade do fenômeno nas diferentes regiões. Para os 496 municípios, o menor índice $(0,165)$ calculado refere-se ao município Passa Sete, localizado na região central do estado. Já o maior índice $(0,667)$ está associado à realidade socioeconômica da capital do estado, o município de Porto Alegre, localizado na região Noroeste do estado.

Tillman, Menezes e Fernandez (2017) argumentam que o desigual padrão de desenvolvimento observado nas diferentes regiões do RS pode ser consequência dos ímpares processo de acumulação de capital físico e humano, pela especialização das atividades produtivas e pelas dinâmicas de crescimento populacional de cada região. Neste sentido, as economias de aglomeração que se instalaram às margens de Porto Alegre podem justificar o desempenho auferido em termos de IQV.

A Figura 2 apresenta os estratos de IQV no estado, de forma a ilustrar que poucos municípios possuem os índices maiores que 0,5 e apenas 1 município teve IQV maior que 0,6.

Figura 2 - Índice de Qualidade de Vida nos municípios do Rio Grande do Sul

0,165 a 0,272

0,272 a 0,306

0,306 a 0,335

0,335 a 0,667

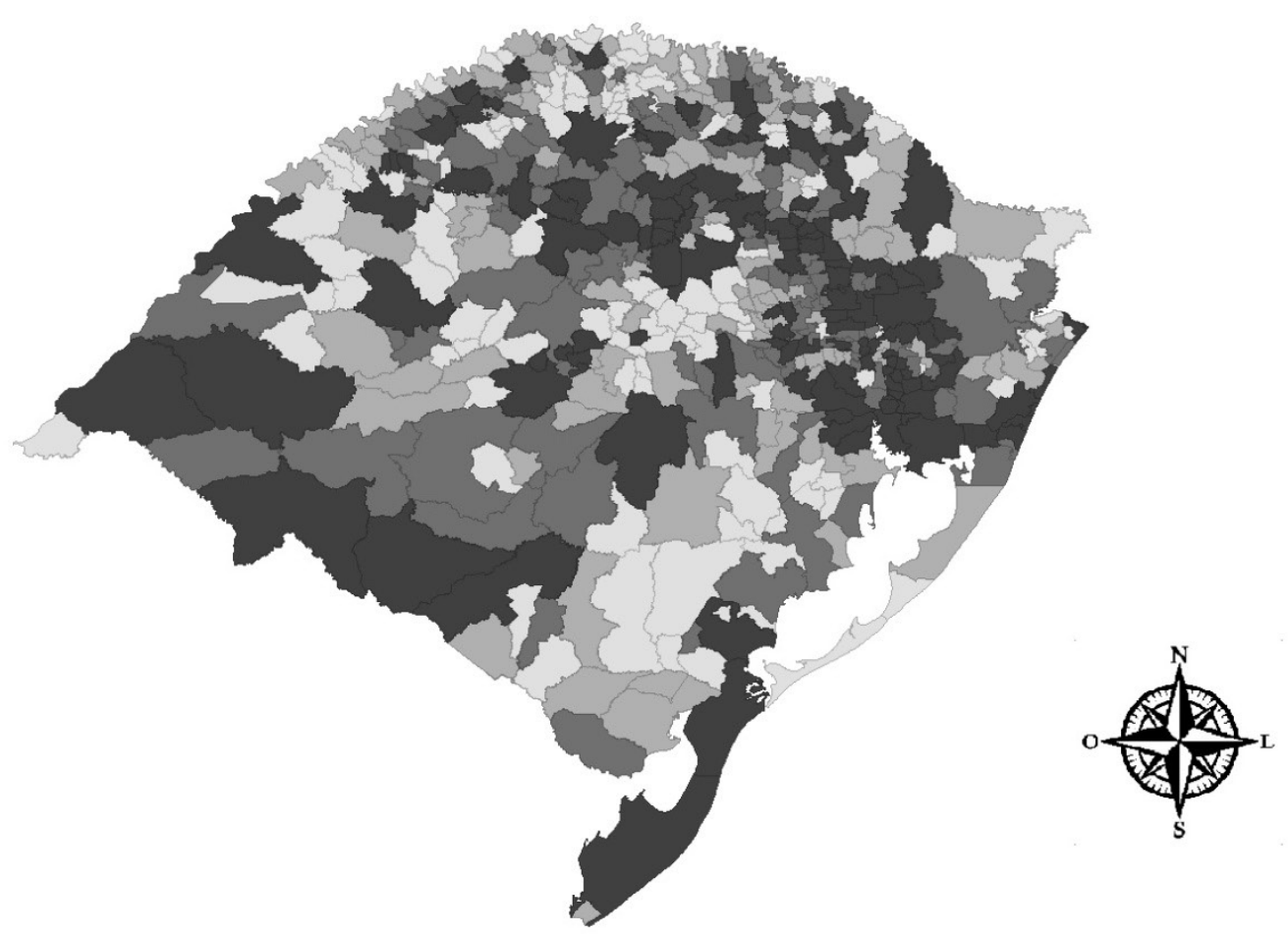

Fonte: Estimativas utilizando dados disponibilizados pelo ADH (2019), IBGE (2019), FEE (2019) e INEP (2019). 
Tendo em vista que há indícios de desigualdades no desempenho observados pelos municípios gaúchos no tocante ao IQV, procede-se com a investigação se os municípios com IQV com baixa qualidade de vida ou alta qualidade de vida estão localizados em uma mesma região do estado. Desse modo, implementou-se a análise exploratória dos dados espaciais, que permitiu a verificação do efeito transbordamento do IQV, podendo assim formar clusters com padrões específicos.

O resultado da I de Moran Global foi de 0,2878, com p-valor de 0,001. Assim sendo, ao nível de significância de $5 \%$, pode-se rejeitar a hipótese nula de que não existe autocorrelação espacial do IQV em 2010. A Figura 3 apresenta o resultado do I de Moran Local. Nota-se que três clusters padrão Alto - Alto e três clusters padrão Baixo - Baixo.

Figura 3 - Mapa de Clusters Univariado do Índice de Qualidade de vida (IQV) em 2010

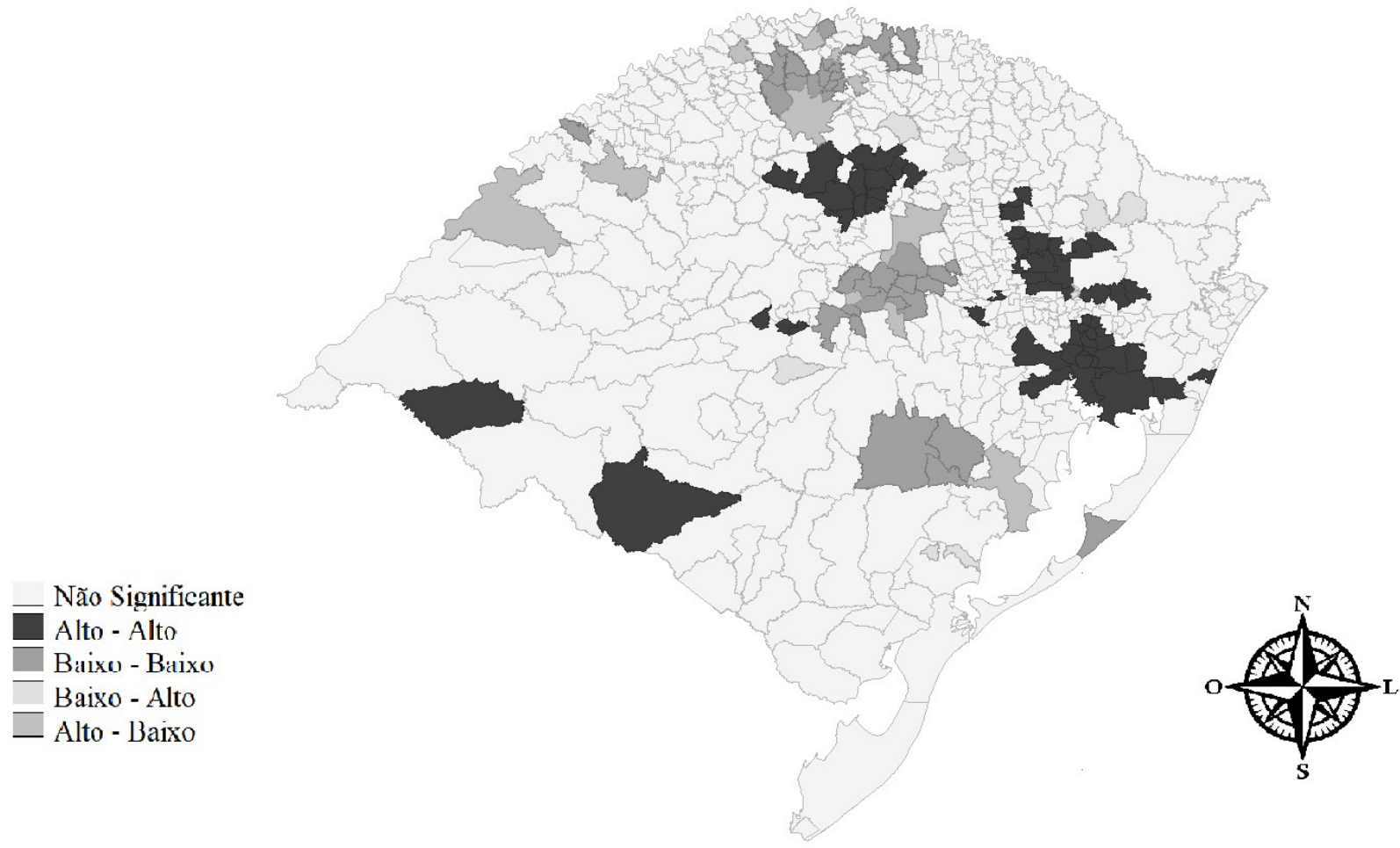

Fonte: Estimativas utilizando dados disponibilizados pelo ADH (2019), IBGE (2019), FEE (2019) e INEP (2019).

Em relação aos clusters padrão Alto - Alto, um deles está situado na região Norte do estado e é composto por municípios como Carazinho (0,389), Santo Antônio do Planalto (0,335), Ernestina (0,311), Não-me-Toque (0,370), Colorado (0,327), Lagoa dos Três Cantos (0,342), Tapera (0,348), Selbach $(0,360)$, Quinze de Novembro $(0,331)$, Ibirubá $(0,371)$, Salvador do Sul $(0,337)$ e Pejuçara (0,346). Outro cluster padrão Alto-Alto está localizado na região Centro-Nordeste e é composto por municípios como Farroupilha $(0,405)$, Flores da Cunha $(0,368)$, Nova Ramada do Sul (0,316), Gramado (0,384). Por fim, o terceiro cluster padrão Alto-Alto está localizado na região Centro-Sul e é composto por municípios como Viamão $(0,396)$, Capivari do Sul $(0,345)$, Porto Alegre $(0,667)$, Alvorada $(0,368)$, Cachoeirinha (0,403), Canoas (0,457), Sapucaia do Sul (0,372), Gravataí (0,411), Glorinha (0,309). Em suma, o padrão Alto - Alto demonstra que um determinado município do cluster com alto IQV é circundado por municípios com alto IQV também.

Os outros três clusters que se formaram são caracterizados pelo padrão Baixo - Baixo, isto é, municípios com Baixo IQV que são circundados por municípios com baixo IQV. Um deles está situado na região Norte do estado, composto por municípios como Erval Seco $(0,260)$, Redentora $(0,174)$, Coronel Bicaco $(0,255)$ e Cerro Grande $(0,245)$. Na região Central do estado também formou-se outro cluster, composto por municípios como Gramado Xavier (0,225), Segredo (0,232), Progresso (0,270), Lagoa Bonita do Sul $(0,232)$ e Agudo $(0,298)$. Outro cluster compreende municípios da região Sul do estado, tais como Encruzilhada do Sul $(0,294)$, Amaral Ferrador $(0,224)$ e Dom Feliciano (0,220).

Esses resultados vão de encontro com os obtidos por Monasterio, Salvo e Damé (2008), onde observou-se a existência de externalidades aglomerativas nas localidades com maior população e maior taxa de urbanização no Rio Grande do Sul, como é o caso da maioria dos municípios que compõem o terceiro cluster Alto-Alto. Ainda de acordo 
com os resultados desse estudo, os clusters industriais e as regiões mais próximas do centro econômico apresentaram diferenciais de salários. Logo, isso também pode explicar parte dos resultados observados dos IQV's nos municípios próximos à capital gaúcha.

De maneira geral, observa-se que o estado do Rio Grande do Sul possui descompassos regionais, caracterizados pelos diferentes desempenhos auferidos pelos municípios no tocante à qualidade de vida da população.Os resultados do desenvolvimento da Análise Fatorial e da Análise Exploratória de Dados Espaciais revelam a importância de políticas públicas para o enfrentamento das principais carências municipais, principalmente naquelas regiões em que o IQV foi considerado baixo. Dado o caráter multidimensional da qualidade de vida, o fomento ao acesso à educação e aos serviços em saúde pode proporcionar melhoria nas condições de vida da população, principalmente à população mais vulnerável e dependente da ação estatal.

Salienta-se que as políticas públicas que fomentam a educação são capazes de expandir as capacidades individuais, além de proporcionar acesso aos postos de trabalho mais remunerados. De fato, vários estudos apontam que as desigualdades no mercado de trabalho são influenciadas pelas características de acesso ao sistema educacional (CAMARANO et al., 2004; CORSEUIL et al., 2001; OSÓRIO, 2004). Logo, ao proporcionar maiores níveis de escolaridade, as políticas são capazes também de fomentar o emprego e, consequentemente, a renda.

Ademais, a população mais escolarizada é, em geral, mais informada, de forma que é possível identificar sinergias positivas sobre a participação dos indivíduos na esfera política, sobre cuidados com a saúde, com a alimentação, entre outras dimensões da vida em sociedade. Portanto, com o acesso à educação, à saúde, ao trabalho e renda, os indivíduos podem alcançar melhorias no que tange ao seu bem-estar.

\section{Considerações finais}

O presente estudo propôs-se a construir um Índice de Qualidade de Vida multidimensional para caracterizar a realidade socioeconômica dos municípios do Rio Grande do Sul. Ferramentais econométricos permitiram a extração de quatro fatores - Indicador de acesso a bens duráveis, Indicador educacional, Indicador da capacidade econômica para obter melhores condições de moradia e Indicador de condições de saúde -, os quais compuseram o IQV final.

Dentre os principais resultados da pesquisa, notou-se que, para o ano de 2010, formaram-se seis clusters no estado, ou seja, alguns municípios são circundados por municípios com o mesmo nível de qualidade de vida. Foi possível identificar que os clusters padrão Alto - Alto estão localizados na região norte, nordeste e centro sul do estado, sugerindo que o nível de desenvolvimento desses municípios é maior, desencadeando transbordamentos sobre o nível de qualidade de vida.

Porém, observa-se que na região norte foi também formado um cluster padrão Baixo - Baixo, que está localizado próximo ao cluster padrão Alto -Alto. Esses resultados apontam para a existência de desequilíbrios intra-regionais. Por sua vez, a região central do estado destacou-se pela existência de dois clusters padrão Baixo - Baixo, apresentando municípios com piores condições econômicas, de saúde, de moradia e educação.

Dado o padrão territorial de distribuição dos IQVs, julga-se importante a ação focalizada na promoção de qualidade de vida para a população mais vulnerável dos municípios gaúchos. Pelo perfil dos resultados econométricos da presente pesquisa, o conjunto de ações públicas pode ser direcionado ao fomento da educação básica de qualidade, ao acesso universal de serviços de saúde e melhoria das condições de moradia.

\section{Referências}

ALONSO, J. A. F.; BENETTI, M. D.; BANDEIRA, P. S. Crescimento econômico da região sul do Rio Grande do Sul: causas e perspectivas. Porto Alegre: Fundação de Economia e Estatística Siegfried Emanuel Heuser, 1994.

ALMEIDA, E. Econometria espacial. Campinas: Alínea, 2012.

AREND, M.; CARIO, S. A. F. Desenvolvimento e desequilíbrio industrial no Rio Grande do Sul: uma análise secular evolucionária. Economia \& Sociedade, Campinas, v. 19, n. 2, p. 381-420, 2010.

BERNARDINI, R.; KANG, T. H.; WINK JR., M. V. Desigualdades regionais no Rio Grande do Sul: uma abordagem multidimensional, utilizando o Índice de Desenvolvimento Socioeconômico (Idese), 2007-2010.

Indicadores Econômicos FEE, Porto Alegre, v. 42, n. 4, p. 59-72, 2015.

CAMARANO, A. A.; MELLO, J. L.; PASINATO, M. T.; KANSO, S. Caminhos para a vida adulta: as múltiplas 
trajetórias dos jovens brasileiros. Rio de Janeiro: IPEA, 2004.

CORSEUIL, C. H.; SANTOS, D. D.; FOGUEL, M. N. Decisões críticas em idades críticas: a escolha dos jovens entre estudo e trabalho no Brasil e em outros países da América Latina. Rio de Janeiro: IPEA, 2001.

CONTERATO, M. A.; SCHNEIDER, S.; WAQUIL, P. D. Desenvolvimento rural no Estado do Rio Grande do Sul: uma análise multidimensional de suas desigualdades regionais. REDES, Santa Cruz do Sul, v. 12, n. 2, p. 163 -195, 2007.

FEE, Fundação de Economia e Estatística. Metodologia Idese - 2015, 2018. Disponível em: <https://www.fee. rs.gov.br/indicadores/indice-de-desenvolvimento-socioeconomico/metodologia/>. Acesso em: 18 set. 2019.

FERREIRA, M. A.; SANTOS, E. C.; LIMA, J. E. Índice de avaliação ambiental: Uma análise a partir de modelos estatísticos multivariados. Revibec: revista de la Red Iberoamericana de Economía Ecológica, v. 26, p. 177-190, 2016.

FIORI, T. P. Desigualdades regionais no Rio Grande do Sul. Carta Conjuntura FFE, v. 21, n. 8, p. 7, 2012.

FREITAS, T. D. A diversificação dos meios de vida como expansão das capacitações : por uma sociologia das condições de vida na fumicultura no Rio Grande do Sul. 2015. 326p. Tese (Doutorado em Sociologia) Universidade Federal do Rio Grande do Sul, Porto Alegre, RS, 2015.

HAUSMAN, D.; MC PHERSON, M.; SATZ, D. Economic analysis, moral philosophy, and public policy. Cambridge: Cambridge University Press, 2016.

IBGE, Instituto Brasileiro de Geografia e Estatística. Censo Demográfico, 2010. Disponível em:< https:// censo2010.ibge.gov.br/resultados.html>. Acesso em: 09 de out. 2019.

INEP, Instituto Nacional de Estudos e Pesquisas Educacionais Anísio Teixeira: Dados abertos, 2019. Disponível em: <http://inep.gov.br/dados>. Acesso em: 08 out 2019.

MARQUETTI, A. A.;BÊRNI, D. A.; MARQUES, A. M. Determinantes dos diferenciais das taxas de crescimento sub-regionais do Rio Grande do Sul nos anos 90. Ensaios FEE, Porto Alegre, v. 26, Número Especial, p. 95-116, 2005.

MINAYO, M. C. S.; HARTZ, Z. M. A.; BUSS, P. M. Qualidade de vida e saúde: um debate necessário. Ciênc. saúde coletiva, Rio de Janeiro, v. 5, n. 1, p. 7-18, 2000.

MINGOTI, S. A. Análise de dados através de métodos de estatística multivariada: uma abordagem aplicada. Belo Horizonte: Editora UFMS, 2005.

MONASTERIO, L. M.; SALVO, M.; DAMÉ, O. M. Estrutura espacial das aglomerações e determinação dos salários industriais no Rio Grande do Sul. Ensaios FEE, Porto Alegre, v. 28, Número Especial, p. 801-824, 2008.

MYRDAL, G. An international economy: problems and prospects. Nueva York: Harper, 1956.

NUSSBAUM, M.; SEN, A. The Quality of Life. Clariton: Paperbacks, 1993.

OLIVEIRA, C. Desigualdades regionais no Rio Grande do Sul: um enfoque da nova geografia econômica. REDES, Santa Cruz do Sul, v. 10, n. 2, p. 93-116, 2005.

OLIVEIRA, L. L. S. Uma década do Índice de Desenvolvimento Socioeconômico (Idese) do Rio Grande do Sul: evolução no período 2000-09. Indicadores Econômicos FEE, Porto Alegre, v. 41, n. 2, p. 119-134, 2013.

OSORIO, R. G. A Mobilidade social dos negros brasileiros. Rio de Janeiro, IPEA, 2004.

PALOMBA, R. Calidad de Vida: Conceptos y medidas. Taller sobre calidad de vida y redes de apoyo de las 
personas adultas mayores. Santiago: CEPAL, 2002.

PAULANI, Leda Maria; BRAGA, Márcio Bobik. A nova contabilidade social. Saraiva, 2007.

PEREIRA, É. F.; TEIXEIRA, C. S.; SANTOS, A. Qualidade de vida: abordagens, conceitos e avaliação. Revista Brasileira de Educação Física e Esporte, São Paulo, v. 26, n. 2, p. 241-250, 2012.

PNUD, Programa das Nações Unidas para o Desenvolvimento; IPEA, Instituto de Pesquisa Econômica Aplicada; FJP, Fundação João Pinheiro. Atlas do desenvolvimento humano no Brasil, 2013. Disponível em: $<$ http:// atlasbrasil.org.br/2013/pt/consulta/>. Acesso em: 02 out. 2019.

SCHNEIDER, S.; FREITAS, T. D. Qualidade de Vida, Diversificação e Desenvolvimento: referências práticas para análise do bem-estar no meio rural. Revista Olhares Sociais. Dossiê Teoria Social e Desenvolvimento, v. 2, n. 1, p. $121-142,2013$.

SEIDL, E. M. F.; ZANNON, C. M. L. C. Qualidade de vida e saúde: aspectos conceituais e metodológicos. Cad. Saúde Pública, Rio de Janeiro, v. 20, n. 2, p. 580-588, 2004.

SEN, A. Desenvolvimento como Liberdade. São Paulo: Cia das Letras, 2001.

TILLMAN, E. A.; MENEZES, G. R.; FERNANDEZ, R. N. Análise das diferenças regionais do estado do Rio Grande do Sul. Revista ESPACIOS, v. 38, n. 32, 2017.

UL HAQ, M. O Paradigma do Desenvolvimento Humano. In: Desenvolvimento Humano: leituras selecionadas do PNUD. Belo Horizonte: PUC Minas Virtual: PNUD, 2007.

VASCONCELOS, M. A.; GARCIA, M. E. Fundamentos de economia. São Paulo: Saraiva, 1998.

VÁZQUEZ, S. T.; MONTELLANO, A. Q.; SOBRAO, D. G.; AGUILAR, R. M. Manuales sobre cooperación y desarrollo Desarrollo: Desarrollo humano, pobreza y desigualdades. Cátedra de Cooperación Internacional y com Iberoamérica, Universidad de Cantabria, 2013.

WAQUIL, P. D.; FILIPPI, E. E. Desigualdades regionais e desempenho diferenciado dos municípios do RS: uma análise a partir de elementos rurais. In: Carlos Águedo Paiva. (Org.). Evolução das desigualdades territoriais no Rio Grande do Sul. Santa Cruz do Sul, RS: EDUNISC, 2008.

\section{Agradecimentos}

As autoras agradecem o apoio do Conselho Nacional de Desenvolvimento Científico e Tecnológico (CNPq) e da Coordenação de Aperfeiçoamento de Pessoal de Nível Superior (Capes) pelo auxílio financeiro para a execução da pesquisa. 